\title{
Escola de pais do Brasil: Análise de perfil e impacto no apoio social, prá- ticas parentais, capacidades e dificuldades dos filhos e estresse parental
}

\author{
Bruna Detoni \\ Adriane Xavier Arteche \\ Adolfo Pizzinato
}

\section{RESUMO}

Escola de Pais do Brasil (EPB) é uma associação voluntária, sem fins lucrativos, filantrópica e educacional. Círculo de Debates, sua principal ação, consiste em uma ação parental de caráter preventivo, realizado em encontros que discutem temas educativos sugeridos pela EPB. A pesquisa pretendeu traçar um perfil dos pais/mães que participaram dos Círculos de Debates no primeiro semestre de 2018 em diferentes cidades gaúchas, e avaliar o impacto dessa participação na rede de apoio, nas práticas parentais, na percepção das capacidades e dificuldades dos/as filhos/as e nos níveis de estresse parental. Realizou-se um estudo quasi-experimental que aplicou um questionário sociodemográfico e instrumentos para investigar as variáveis em participantes dos Círculos de cinco municípios, tomados como população da amostra. Foram feitas análises descritivas e análises de medidas repetidas. A maioria dos participantes foi composta de mulheres, de escolaridade superior, com um filho entre 7 e 12 anos. Concluída a ação, verificou-se que aumentou significativamente a participação comunitária e o envolvimento em questões relacionadas à disciplina. Também diminuíram a percepção de sintomas emocionais, problemas de comportamento e hiperatividade. As análises possibilitaram inferir que o trabalho do Círculo de Debates tem um impacto positivo no exercício da parentalidade.

Palavras-chave: parentalidade; sintomas emocionais; apoio social.

\section{ABSTRACT}

Parents school of Brazil: Analysis of profile and impact on social support, parental practices, perceptions of capacities and grades of children and parental stress

Escola de Pais do Brasil (EPB) is a voluntary, non-profit, philanthropic, and educational association. Círculo de Debates, its main action, consists of parental action of a preventive nature, held in meetings that discuss educational topics suggested by the EPB. This research aims to draw a profile of parents who participated in these Círculo de Debates in the first semester of 2018 in different cities in Rio Grande do Sul, and to evaluate the impact of this participation on the support network, parental practices, perception of abilities and difficulties of children, and levels of parental stress. A quasi-experimental study was conducted that applied a sociodemographic questionnaire and instruments to investigate the variables in Circle participants from five municipalities, taken as the sample population. Descriptive analyses and repeated measures analyses were performed. The majority of participants were women, with higher education, and with one child between 7 and 12 years old. At the conclusion of the action, it was found that community participation and involvement in discipline-related issues increased significantly. They also decreased the perception of emotional symptoms, behavior problems, and hyperactivity. The analysis made it possible to infer that the work of the Debate Circle has a positive impact on the exercise of parenting.

Keywords: parenting; emotional symptoms; social support.

\section{Sobre os autores \\ B. D. \\ https://orcid.org/0000-0002 \\ 6127-4625 \\ Pontifícia Universidade Católica do Rio Grande do Sul - Porto Alegre, RS \\ psibrunadetoni@gmail.com}

\section{A. X. A.}

https://orcid.org/0000-0002-

2586-4900

Pontifícia Universidade Católica do Rio Grande do Sul - Porto Alegre, RS

adriane.arteche@pucrs.br

\section{A. P.}

https://orcid.org/0000-0002-

1777-5860

Universidade Federal do Rio

Grande do Sul - Porto Alegre, RS adolfopizzinato@hotmail.com

\section{Direitos Autorais}

Este é um artigo de acesso aberto e pode ser reproduzido livremente, distribuído, transmitido ou modificado, por qualquer pessoa desde que usado sem fins comerciais. 0 trabalho é disponibilizado sob a licença Creative Commons CC-BY-NC.

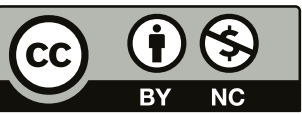




\section{H. INTERACÃO EM PSICOLOGIA}

A parentalidade é definida como o conjunto de atividades que têm o propósito de favorecer a sobrevivência e o desenvolvimento da criança num ambiente seguro, de modo a socializá-la e torná-la progressivamente mais autônoma (Barroso \& Machado, 2010). É descrita como uma das tarefas mais complexas, difíceis e com maiores desafios e responsabilidades para o ser humano. Segundo Houzel (2004), esse conceito difere do conceito legal de genitor, uma vez que não basta ser genitor nem ser designado como pai para preencher todas as condições de parentalidade, é necessário "tornar-se pai", o que acontece por meio de um processo complexo, implicando diferentes níveis de funcionamento social, psicológico e cultural.

Belsky (1984), já nos anos 1980, propõe um modelo ecológico dos determinantes da parentalidade, afirmando que a funcionalidade parental é composta por múltiplos fatores que integram três instâncias principais: 1) as características dos pais/mães/cuidadores; 2) as características dos filhos/filhas; e 3) as características do contexto social. Dessa forma, a história de desenvolvimento dos pais, as relações conjugais, as redes sociais e o trabalho atuam sobre a personalidade individual e o bem-estar psicológico geral dos pais, contribuindo para a funcionalidade parental, com consequências para o desenvolvimento da criança. Para o autor, o contexto social e as fontes de estresse influenciam direta ou indiretamente a funcionalidade da parentalidade.

De acordo com Maccoby (2000), as práticas educativas têm grande peso no ajustamento psicológico dos filhos. Para compreendermos as problemáticas que podem surgir na interação entre a criança e seus cuidadores, é necessário reconhecer o impacto das dificuldades parentais em providenciar à criança cuidado, conforto, disponibilidade e orientação (Barroso \& Machado, 2010). Por isso, é importante conhecer como se dá a relação e interação dos cuidadores com seus/ suas filhos/as.

As práticas parentais incluem ações, técnicas e métodos específicos para ensinar à criança um determinado valor ou chamar sua atenção para que possa adotar ou corrigir atitudes ou comportamentos (Macana \& Comin, 2015). Considerando a funcionalidade das práticas parentais, elas podem ser positivas ou negativas. Quando são positivas, favorecem o desenvolvimento cognitivo e socioemocional das crianças. As que mais se destacam são o comportamento moral, as expressões afetivas, o envolvimento dos pais no brincar, o reforço e a disciplina. Apesar de a identificação das práticas parentais negativas e o mecanismo de seus efeitos na infância serem temas muito mais complexos, Macana e Comim (2015) apresentam um resumo das que são destacadas pela literatura, como o abuso físico e psicológico, a disciplina relaxada, a disciplina coercitiva, a punição inconsistente, a monitoria estressante e a comunicação negativa.
Bruna Detoni, Adriane Xavier Arteche e Adolfo Pizzinato

Além das práticas educativas, as redes sociais têm um papel importante na vinculação das famílias no processo de desenvolvimento de habilidades que auxiliem e fortaleçam seu potencial para o desenvolvimento infantil saudável em todas as suas dimensões. A rede social de apoio familiar é composta pela família nuclear, mães, pais, avós, tios, irmãos, e também pessoas que integram a rede expandida, com a qual a criança estabelece relações. Compreender os elementos que compõem essa rede pode ajudar a estabelecer ações que promovem o desenvolvimento infantil (Dezoti et al., 2013).

Em uma revisão sistemática sobre estresse parental, Brito e Faro (2016) constataram estressores presentes no contexto parental, principalmente referidos às características dos filhos. Algumas variáveis sociodemográficas, como renda familiar, escolaridade dos pais e número de filhos, podem influenciar o nível de estresse parental. Além disso, o suporte social recebido costuma auxiliar pais e mães a lidar de forma adaptativa com a sobrecarga de estresse, ou seja, quanto maior o suporte social e a satisfação com o apoio recebido, menor o estresse parental. Os autores apresentaram ainda evidências a respeito da relação entre estresse parental e práticas parentais. Estas, quando negativas, parecem comprometer não só o desenvolvimento da criança e a interação familiar, mas também a saúde dos pais, uma vez que o estresse parental surge como produto desse processo.

Diversos são os programas de intervenção para pais e mães, destinados a situações de desenvolvimento não normativo ou a famílias em situação de vulnerabilidade que almejam fomentar práticas parentais mais positivas. Desenvolvido em Portugal, destaca-se o programa PApi - Pais por inteiro, para desenvolver o ajustamento pessoal e promover a coparentalidade positiva com pais divorciados (Lamela et al., 2012) e o Programa Parentalidade Positiva, da Associação Nacional de Estudo e Intervenção em Sobredotação, que visa promover a parentalidade positiva de pais de crianças sobredotadas (Pinho-Pereira et al., 2017).

$\mathrm{Na}$ Espanha, há o Programa Crecer Felices en la familia, implementado por serviços sociais locais e organizações não governamentais, voltado para pais em situação de risco psicossocial com filhos entre zero e cinco anos de idade (Álvarez et al., 2018). Tal programa foi mencionado por Rodrigo (2016), em pesquisa sobre a qualidade da implementação de programas para o desenvolvimento de práticas parentais positivas na Espanha, baseado em evidências. A pesquisa refere ainda outros programas e seus objetivos, como o Programa Formación y Apoyo Familiar, destinado a pais de crianças de qualquer faixa etária em situações de risco psicossocial; o Programa Aprender Juntos e Crecer Felices en la Familia, voltado para pais em situações de risco e crianças entre seis e 11 anos, com foco preventivo de promoção de relações positivas entre pais e filhos; o Programa de Competencia Familiar, voltado 


\section{4* INTERACÃO EM PSICOLOGIA}

para prevenção de risco de famílias que estão em tratamento de reabilitação do uso de drogas; o Programa Vivir la Adolescencia en Familia, para pais em situações de risco psicossocial e que tenham filhos adolescentes; o Programa-Guía para el Desarollo de Competencias Emocionales, que promove competências parentais que dão suporte às práticas parentais positivas de pais com filhos em qualquer faixa etária, visando também à prevenção de conflitos familiares; e o programa Educar en Positivo, que oferece suporte online para ajudar os pais a melhorarem suas habilidades parentais e aprenderem mais sobre educação infantil e questões familiares.

Esses programas foram desenvolvidos na própria Espanha ou adaptados de outras culturas e, em sua maioria, recebem apoio financeiro público ou privado para seu desenvolvimento, implementação e avaliação. Os facilitadores são profissionais que trabalham em serviços públicos ou privados, treinados pelas equipes de cada programa. Além disso, as iniciativas relacionadas ao desenvolvimento desses trabalhos são influenciadas pelas recomendações do Conselho da Europa sobre Políticas para dar suporte às práticas parentais positivas. Entre os aspectos valorizados na pesquisa de Rodrigo (2016), destaca-se o impacto das ações nas mudanças dos estilos e no desenvolvimento de competências parentais. Outros destaques importantes dizem respeito ao papel crucial desempenhado pelos facilitadores e à necessidade de atenção às diferenças dos contextos na adaptação dos programas.

Schmidt et al. (2016), em uma revisão integrativa sobre a promoção de intervenções de práticas parentais positivas, destacaram três programas de intervenção que contribuem para a qualidade das práticas parentais e, consequentemente, favorecem o desenvolvimento infantil: Triple $P-P$ sitive Parenting Program (Austrália), Incredible Years (EUA) e Parent Managment Training (EUA). Os estudos evidenciaram impactos significativos na melhoria da satisfação parental, da eficácia parental e das relações pais-criança. Além disso, constataram que a intervenção auxiliou os participantes a fortalecer a identidade parental e a conectividade na relação pais-criança, além de identificar a influência das histórias de vida de cada um nas práticas parentais adotadas e no desenvolvimento infantil. Esse efeito parece ter sido fomentado pelas reflexões em grupo (To et al., 2014), que favoreceram o aumento do senso de competência e a redução do estresse parental (Schmidt et al. 2016; Guisso et al., 2019). A despeito da origem, tais programas acontecem em vários países, tanto para a população em geral como para a população com necessidades específicas, pautados na evidência de que, ao meIhorar a qualidade das práticas parentais, ocorre a promoção de um funcionamento infantil mais saudável.

No Brasil, existem alguns serviços públicos de saúde e assistência voltados às famílias. Na área da saúde, há o Primei-
Bruna Detoni, Adriane Xavier Arteche e Adolfo Pizzinato

ra Infância Melhor (PIM) e o Programa de Saúde da Família (PSF); na área da assistência, o Programa Criança Feliz (PCF) e ações do Centro de Referência de Assistência Social (CRAS), no contexto da assistência básica, e do Centro de Referência Especializado de Assistência Social (CREAS), voltados a uma parcela da população com direitos violados. Especificamente para a promoção de práticas parentais positivas, apesar da ampliação de programas dessa natureza, ainda existem poucos programas, e os programas desenvolvidos, apesar de serem avaliados positivamente, podem ser considerados como tendo baixa adesão dos participantes, conforme referem Guisso et al. (2019), após revisão sistemática de práticas parentais e de treinamento parental. Um exemplo é o Programa Qualidade na Interação Familiar (PQFI), que orienta e treina pais para aprenderem a manejar diferentes situações de práticas educativas, composto de oito encontros constituídos de vivências, discussões, explicações teóricas, treinamento prático em casa e auto registro (Weber et al., 2006).

Diante da carência de estudos nacionais e internacionais sobre tal temática, destaca-se as ações da Escola de Pais do Brasil (EPB), que oferece o Círculo de Debates, composto de sete encontros semanais, com assuntos pré-determinados. Seu objetivo é discutir com pais/mães e educadores de crianças e adolescentes as necessidades, dificuldades, problemáticas e possíveis alternativas para melhor entender as situações cotidianas relacionadas aos seus/suas filhos/as e exercitar a parentalidade. Ultimamente, alguns casais grávidos ou tentantes também têm participado. Atualmente, cada encontro desenvolve uma temática diferente: 1) Educar: um desafio; 2) Valores e limites na educação dos filhos; 3) Pai, mãe e agentes educadores; 4) A educação do nascimento à puberdade; 5) Adolescência: o segundo nascimento; 6) Sexualidade no ciclo da família e da vida; e 7) Cidadania e cultura da paz (Escola de Pais do Brasil, 2021). Os Círculos são coordenados por um casal facilitador, associado à EPB, que já participou de outros eventos semelhantes e recebeu um treinamento para se tornar facilitador. A EPB realiza esse trabalho há mais de 50 anos em diversas cidades do Brasil, porém não há pesquisas ou publicações a respeito da eficácia da ação, nem avaliações sobre a qualidade do auxílio proporcionado a pais/mães/cuidadores no exercício de práticas parentais mais positivas.

Assim, o presente trabalho descreve o perfil dos pais/ mães que participaram dos Círculos de Debates, entre março e julho de 2018, em cinco municípios do Rio Grande do Sul, e avalia o impacto dessa participação. Para tanto, os pais/mães foram avaliados em suas estratégias de apoio social, práticas parentais, percepção acerca das capacidades e dificuldades dos/as filhos/as e níveis de estresse parental antes e depois de terem participado dos encontros, com vistas a testar a hipótese de que passariam a ter práticas parentais mais posi- 
tivas, percebendo melhor as capacidades e dificuldades dos filhos, diminuindo o estresse parental e aumentando a rede de apoio social.

\section{MÉTODO}

\section{DELINEAMENTO}

Foi realizado um estudo quasi-experimental, no período de março a julho de 2018, mediante a aplicação de um questionário sociodemográfico e de instrumentos capazes de identificar a rede e as estratégias de apoio social e comunitário, as práticas parentais, a percepção das capacidades e dificuldades dos/as filhos/as e os níveis de estresse parental, antes (T1) e depois (T2) da participação de pais/cuidadores no Círculo de Debates da EPB. O corpus de análise foi composto por cidades gaúchas que aderiram voluntariamente ao estudo: Campinas do Sul (Seccional Erechim), Carazinho, Casca (Seccional Marau), Erechim e Gramado.

\section{PARTICIPANTES}

A amostra final deste estudo abrangeu 104 pais/mães/ cuidadores que tinham filhos e participaram do Círculo de Debates da EPB. 0 critério de inclusão para a análise descritiva foi a) participar dos encontros da EPB, tendo recebido o certificado de conclusão, fornecido aos que compareceram a, no mínimo, cinco encontros. Para análise de efeito da ação, considerou-se ainda a necessidade de b) ter pelo menos um filho biológico ou adotivo. 0 critério de exclusão foi ter respondido os instrumentos T1 e T2 sobre filhos diferentes.

Inicialmente, 164 participantes aceitaram participar do estudo, responderam ao termo de consentimento livre e esclarecido e os instrumentos no T1. No entanto, 60 foram excluídos por não terem recebido o certificado de conclusão do Círculo e/ou não terem respondido os instrumentos $\mathrm{T} 1 \mathrm{e} \mathrm{T} 2$ a respeito do mesmo filho. Uma amostra de 104 participantes foi considerada para a descrição do perfil e escores do Questionário de Apoio Social/Comunitário (QASC). Para as demais análises, foi considerado uma amostra de 99 participantes (98 pais/ mães e 1 cuidador), uma vez que outros cinco não compuseram os demais escores por ainda não terem filhos. A Tabela 1 apresenta as características demográficas da amostra.

\section{INSTRUMENTOS}

Questionário de características sociodemográficas: contém informações como idade, sexo, estado civil, escolaridade, profissão, ocupação, quantidade de filhos, idade dos filhos, com quem mora, com quem moram os filhos e outros dados relevantes para o estudo.
Tabela 1. Dados sociodemográficos da amostra da $E P B$

\begin{tabular}{|c|c|}
\hline Característica & $\mathrm{n}(\%)$ \\
\hline \multicolumn{2}{|l|}{ Cidade $(n=104)$} \\
\hline Carazinho & $13(12,5 \%)$ \\
\hline Casca & $25(24 \%)$ \\
\hline Erechim & $26(25 \%)$ \\
\hline Gramado & $2(1,9 \%)$ \\
\hline Campinas do Sul & $38(36,5 \%)$ \\
\hline \multicolumn{2}{|l|}{ Sexo $(n=104)$} \\
\hline Masculino & $42(40,4 \%)$ \\
\hline Feminino & $62(59,6 \%)$ \\
\hline \multicolumn{2}{|l|}{ Estado Civil $(n=102)$} \\
\hline Com companheiro(a) & $98(96,1 \%)$ \\
\hline Sem companheiro(a) & $4(3,9 \%)$ \\
\hline \multicolumn{2}{|c|}{ Participando com companheiro(a) $(n=101)$} \\
\hline $\operatorname{Sim}$ & $80(79,2 \%)$ \\
\hline Não & $21(20,8 \%)$ \\
\hline \multicolumn{2}{|l|}{ Possui religião (n=104) } \\
\hline $\operatorname{sim}$ & $100(96,2 \%)$ \\
\hline Não & $4(3,8 \%)$ \\
\hline \multicolumn{2}{|l|}{ Escolaridade $(n=104)$} \\
\hline Ensino Fundamental & $22(21,2 \%)$ \\
\hline Ensino Médio & $29(27,9 \%)$ \\
\hline Ensino Superior & $53(51 \%)$ \\
\hline \multicolumn{2}{|l|}{ Renda Familiar $(n=99)$} \\
\hline Até $\mathrm{R} \$ 5000,00$ & $53(53,5 \%)$ \\
\hline Entre $R \$ 5000,00$ e $R \$ 10000,00$ & $34(34,3 \%)$ \\
\hline Acima de $\mathrm{R} \$ 10000,00$ & $12(12,1 \%)$ \\
\hline \multicolumn{2}{|l|}{ Quantas horas trabalha por dia $(n=97)$} \\
\hline Menos de 8 horas & $17(17,5 \%)$ \\
\hline De 8 a 12horas & $72(74,2 \%)$ \\
\hline Mais de 12 horas & $8(8,2 \%)$ \\
\hline \multicolumn{2}{|l|}{ Tem filhos $(n=104)$} \\
\hline $\operatorname{Sim}$ & $98(94,2 \%)$ \\
\hline Não & $6(5,8 \%)$ \\
\hline \multicolumn{2}{|l|}{ Quantos filhos (n=98) } \\
\hline 1 & $50(51 \%)$ \\
\hline 2 & $40(40,8 \%)$ \\
\hline 3 & $7(7,1 \%)$ \\
\hline 4 & $1(1 \%)$ \\
\hline \multicolumn{2}{|l|}{ Faixa etária dos filhos $(n=99)$} \\
\hline 0 a 3 anos & $22(22,2 \%)$ \\
\hline 4 a 6 anos & $24(24,2 \%)$ \\
\hline 7 a 12 anos & $27(27,3 \%)$ \\
\hline 13 a 18 anos & $16(16,2 \%)$ \\
\hline Acima de 19 anos & $10(10,1 \%)$ \\
\hline \multicolumn{2}{|l|}{ Tem netos? $(n=86)$} \\
\hline $\operatorname{Sim}$ & $5(5,8 \%)$ \\
\hline Não & $81(94,2 \%)$ \\
\hline
\end{tabular}




\section{MLE INTERACÃO EM PSICOLOGIA}

Questionário de Apoio Social / Comunitário: criado por Gracia et al. (2002), avalia a participação e integração comunitária (escala 1), a percepção de apoio social em sistemas formais (escala 2) e informais (escala 3), por meio de 25 afirmações a serem respondidas em uma escala tipo Likert de cinco pontos. As três escalas consideradas neste estudo e os valores de fidedignidade da validação original (coeficiente alfa) para cada escala são: $0.879,0.856$ e 0.845 .

Inventário de Práticas Parentais (IPP): criado por Benetti e Balbinotti (2003), foi construído para avaliar as práticas parentais, considerando o envolvimento afetivo, a didática (educação), a disciplina, os aspectos sociais e a responsabilidade do envolvimento parental. O Inventário completo possui 29 itens de avaliação, constituídos de frases afırmativas a serem respondidas numa escala tipo Likert de cinco pontos. Os coeficientes alfa no estudo de validação variam de 0,55 a 0,82 (Grzybowski \& Wagner, 2010). Foram considerados para este estudo os eixos Envolvimento Social, Envolvimento Didático, Envolvimento com a Disciplina, Envolvimento Afetivo e Responsabilidade, além do escore total.

Questionário de Capacidades e Dificuldades (SDQ-Por): criado por Robert Goodman em 1997, é um questionário de rastreamento de problemas de saúde mental, que avalia o comportamento de crianças e adolescentes dos 4 aos 16 anos. É composto por 25 itens em uma escala Likert de três pontos, sendo cinco itens sobre capacidades, 19 itens sobre dificuldades e um item neutro. Está dividido em cinco subescalas, cada uma com cinco afırmações. A versão utilizada para este estudo foi a respondida pelos pais. Para o índice de consistência interna, o alfa de Cronbach apresentou, para as três versões utilizadas, valores próximos de 0,80 (Saur \& Loureiro, 2012). Para as análises deste estudo, foram consideradas as subescalas: Sintomas Emocionais (cujo escore pode ser classificado em normal: 0-3 pontos, limítrofe: 4 pontos, anormal: 5-10 pontos), Problemas de Conduta (normal: 0-2, limítrofe: 3, anormal: 4-10), Hiperatividade (normal: 0-5, limítrofe: 6 , anormal: 7-10), Problemas de relacionamento com os colegas (normal: 0-2, limítrofe: 3, anormal: 4-10), Comportamento pró-social (normal: 6-10, limítrofe: 5, anormal: 0-4) e o escore total (normal 0-13, limítrofe: 14-16, anormal: 17-40), obtido pela soma dos escores nas subescalas Sintomas Emocionais, Problemas de Conduta, Hiperatividade e Problemas de relacionamento com os colegas.

Índice de Estresse Parental (IEP): instrumento americano, criado por Richard Abdin, traduzido para o português e validado para uso no Brasil por Pereira et al. (2016). Trata-se de um questionário de 36 itens, divididos em três domínios - Distúrbio Parental (PD), Interação Disfuncional Parental-Infantil (P-CDI) e Criança Difícil (DC) - combinados para formar uma Escala de Estresse Total. Para obter o valor de cada item, as respostas são dadas por escala Likert de cinco pontos. De
Bruna Detoni, Adriane Xavier Arteche e Adolfo Pizzinato

acordo com Pereira et al. (2016), o valor de confiabilidade de alfa de Cronbach encontrado para o instrumento foi de 0,91, semelhante aos dados do instrumento original. Foram analisados os três domínios, além do escore total de estresse. Os valores considerados como estresse normal são as pontuações entre 16 e 84 . 0 estresse elevado teria pontuações entre 85 e 89 pontos, e o estresse clinicamente significativo, acima de 90 pontos.

\section{RESULTADOS}

\section{PLANO DE ANÁLISE DOS DADOS}

Inicialmente foram realizadas análises descritivas de todos os instrumentos. A seguir, as séries de análises de variância, a fim de investigar o efeito das variáveis demográficas cidade, idade dos pais, sexo dos pais, escolaridade dos pais, faixa etária dos filhos, sexo dos filhos, nos escores de apoio social, práticas parentais, percepção de capacidades e dificuldades dos filhos e níveis de estresse parental do T1. Em um terceiro momento, foram realizadas análises de medidas repetidas, tendo como variáveis dependentes os escores de QASC, IPP, SDQ e IEP. Foram utilizadas como covariáveis as variáveis demográficas com significativa associação com os desfechos. Por fim, foram conduzidas análises complementares para investigar possíveis associações entre as mudanças observadas. Para tanto, foram computados os escores de mudança (T2-T1) e conduzidas análises de correlação entre eles.

\section{EFEITO DAS VARIÁVEIS DEMOGRÁFICAS EM OASC, IPP, SDQ E IEP}

Em relação ao QASC, observou-se um efeito significativo da escolaridade parental no Apoio Social em Sistemas Informais $\left[F(2,86)=4.42, p=.015, \eta^{2}=.09\right]$, sendo que pais com mais baixa escolaridade tiveram menores escores do que pais com mais alta escolaridade [M EF = 32.27, dp=6.83; $\mathrm{M} \mathrm{EM} \mathrm{=}$ 38.37, $\mathrm{dp}=5.31]$.

A variável cidade apresentou diferenças significativas em três escalas desse questionário: Apoio Social em Sistemas Informais $\left[F(4,84)=4.64, p=.002, \eta^{2}=.181\right]$, Integração Comunitária $\left[F(4,84)=4.54, p=.002, \eta^{2}=.178\right]$ e Participação Comunitária $\left[\mathrm{F}(4,84)=18.72, \mathrm{n}=.000, \eta^{2}=.471\right]$. Em relação ao apoio social em sistemas informais, os pais dos municípios de Gramado $(M=27.50, d p=3.90)$ e Erechim $(M=33.78, d p=1.15)$ apresentaram menores escores em relação aos pais de Campinas do Sul $(M=39.07, d p=1.02)$. No que diz respeito ao item integração comunitária, os participantes dos municípios de Carazinho ( $M=15.73$, dp=2.94) e Erechim ( $M=16.43$, dp=3.50) apresentaram menores escores em relação ao município de Casca $(M=19.37, d p=2.96)$. Já no item participação comunitária, os pais dos municípios de Gramado $(M=12, d p=2.83)$, Erechim 


\section{INTERACÃO EM PSICOLOGIA}

$(M=15.84, d p=3.95)$ e Carazinho $(M=16.72, d p=4.36)$ apresentaram menores escores em relação aos municípios de Casca $(M=20.95, d p=3.82)$ e Campinas do Sul $(M=23.10, d p=3.09)$.

Considerando o IPP, houve diferenças significativas relacionadas ao sexo dos pais, e as mães obtiveram maiores escores nos Eixos Envolvimento Didático $[F(1,97)=13.45, p=$ $.000, \eta^{2}=.12, \mathrm{M}$ mãe $=23.84, \mathrm{dp}=4.35, \mathrm{M}$ pai $\left.=20.52, \mathrm{dp}=4.51\right]$, Envolvimento Afetivo $\left[F(1,96)=4.46, p=.037, \eta^{2}=.04, M\right.$ mãe $=35.74, d p=5.28, \mathrm{M}$ pai $=33.38, d p=5.60]$ e Responsabilidade $\left[F(1,96)=12.05, p=.001, \eta^{2}=.11, M\right.$ mãe $=17.86, d p=2.75$, $M$ pai $=15.61, d p=3.64]$. As mães também obtiveram maiores escores no IPP Total $\left[F(1,97)=9.36, p=.003, \eta^{2}=.08, M\right.$ mãe $=115.64, \mathrm{dp}=15.09, \mathrm{M}$ pai $=105.52, \mathrm{dp}=17.60]$.

Entre cidades, foi observada diferença significativa no Eixo Responsabilidade $\left[F(4,93)=2.63, p=.039, \eta^{2}=.10\right]$, sendo que os pais da cidade de Erechim $(M=18.41, d p=2.37)$ apresentaram maiores escores que os pais da cidade de Campinas do Sul $(M=15.79, d p=3.93)$. Além disso, foram constatadas diferenças significativas no Eixo Envolvimento Social, sendo que os pais do município de Erechim apresentaram maiores escores em relação aos pais do município de Casca $[F(4,94)=$ $3.17, p=.017, \eta^{2}=.11, M$ Erechim $=23.00, d p=2.87, M$ Casca $=19.56, d p=3.40]$. Nesse mesmo eixo, pais de filhos na faixa etária de 0 a 3 anos $(\mathrm{M}=22.95, \mathrm{dp}=2.25)$ apresentaram maiores escores do que pais com filhos na faixa etária de 13 a 18 anos $\left[M=18.18, d p=2.99, F(4,94)=4.67, p=.002, \eta^{2}=.16\right]$.

Com relação ao Eixo Envolvimento com a disciplina, pais de escolaridade fundamental apresentaram menor envolvimento do que pais com escolaridade superior $[F(2,95)=5.15, p=$ $\left..007, \eta^{2}=.098, \mathrm{M} \mathrm{EF}=14.71, \mathrm{dp}=5.31, \mathrm{M} \mathrm{ES}=18.00, \mathrm{dp}=3.35\right]$, e pais de crianças entre 0 e 3 anos apresentaram maior envolvimento que pais de crianças entre 7 e 12 anos $[F(4,93)$ $=2.89, \mathrm{p}=.026, \eta^{2}=.11, \mathrm{M} \mathrm{0}-3=19.40, \mathrm{dp}=3.12, \mathrm{M} \mathrm{7-12=}$ $15.96, \mathrm{dp}=4.33$ ]. Observou-se ainda diferenças significativas relacionadas à escolaridade dos pais no Eixo Envolvimento Afetivo $\left[F(2,95)=4.04, p=.021, \eta^{2}=.079, M E F=31.95, d p=7.60\right.$; $\mathrm{MES}=35.69, \mathrm{dp}=4.81]$ e no escore total do $\operatorname{IPP}[F(2,96)=3.96$, $\mathrm{p}=.022, \eta^{2}=.07, \mathrm{M} E F=102.90, \mathrm{dp}=21.43 ; \mathrm{M} \mathrm{EM}=114.25$, $d p=11.74 ; M E S=113.90, d p=15.89]$. Os pais com escolaridade mais alta demonstraram maior envolvimento afetivo e geral em relação aos pais de escolaridade mais baixa.

Em relação ao SDQ, o sexo e escolaridade dos pais influenciaram significativamente a percepção a respeito dos sintomas emocionais que os filhos apresentam. Os pais relataram a presença de mais sintomas emocionais do que as mães $[F(1,92)$ $=4.43, \mathrm{p}=.038, \eta^{2}=.046, \mathrm{M}$ dos pais $=3.52, \mathrm{dp}=2.02 \mathrm{e} \mathrm{M}$ das mãe $=2.67, d p=1.84]$, e houve maior percepção por parte dos pais com escolaridade mais baixa em relação aos com escolaridade mais alta $\left[F(2,91)=4.86, p=.010, \eta^{2}=.097, M E F=3.78\right.$, $\mathrm{dp}=2.14$ e $\mathrm{M} \mathrm{ES}=2.44, \mathrm{dp}=1.69]$. Os sintomas de hiperatividade indicaram diferença significativa em função da faixa etária dos
Bruna Detoni, Adriane Xavier Arteche e Adolfo Pizzinato

pais $\left[F(1,92)=9.90, p=.002, \eta^{2}=.097\right]$, pais mais jovens identificaram mais sintomas de hiperatividade (25-40 anos: $M=4.26$, $d p=2.62$; 41-74 anos: $M=2.62, d p=2.20$ ), e do sexo dos filhos $\left[F(1,93)=5.06, p=.027, \eta^{2}=.052\right]$, evidenciando que os meninos são percebidos como mais hiperativos $(M=4.28, d p=2.55)$ do que as meninas $(M=3.11, d p=2.49)$. A escolaridade dos pais também apontou uma diferença significativa na percepção dos problemas dos filhos com os colegas $[F(2,91)=7.71, p=$ $\left..001, \eta^{2}=.145\right]$, tendo sido referidos mais problemas pelos pais com menor escolaridade $(\mathrm{M} \mathrm{EF}=2.63, \mathrm{dp}=1.70 ; \mathrm{M} \mathrm{EM}=1.50$, $\mathrm{dp}=1.67 ; \mathrm{M} \mathrm{ES}=1.12, \mathrm{dp}=1.12$ ].

No que diz respeito ao IEP, no domínio Distúrbio Parental, a amostra apresentou diferenças significativas entre as cidades de Casca e Erechim, evidenciando um maior estresse nos pais habitantes de Erechim $\left[F(3,84)=3.02, p=.034, \eta^{2}=.097, M\right.$ Casca $=25.46, d p=6.90$ e M Erechim $=30.63, d p=6.12]$. Comparados com as mães $(M=29.19, d p=6.80)-[F(1,86)=5.46$, $\left.p=.037, \eta^{2}=.06\right]$, os pais $(M=25.97, d p=5.63)$ apresentaram menor nível de estresse nesse domínio.

O domínio Interação Disfuncional Parental Infantil sofreu efeito significativo das variáveis cidade $[F(3,84)=2.92, p=$ $\left..039, \eta^{2}=.094\right]$, faixa etária dos filhos $[F(4,83)=7.03, p=.000$, $\left.\eta^{2}=.253\right]$ e escolaridade dos pais $\left[F(2,85)=7.3, p=.001, \eta^{2}=\right.$ .147], ou seja, pais da cidade de Carazinho $(M=27.30, d p=9.02)$ apresentaram maior nível de estresse do que pais da cidade de Erechim $(M=20.90, d p=5.48)$, pais de crianças na faixa etária entre 0 e 3 anos $(M=18.18, d p=5.63)$ apresentaram menores níveis de estresse do que pais de crianças nas faixas etárias entre 7 e 12 anos $(M=26.20, d p=8.22)$ e entre 13 e 18 anos $(M=27.50, d p=6.41)$, e pais com escolaridade superior $(M=20.70, d p=6.20)$ apresentaram menores níveis de estresse do que pais com escolaridade média $(M=26.28, d p=6.90)$ e com escolaridade fundamental $(M=25.87, d p=7.48)$.

O domínio Criança Difícil apresentou diferenças significativas entre cidades $\left[F(3,84)=2.92, p=.039, \eta^{2}=.094\right]$ e faixa etária dos filhos $\left[F(4,83)=3.92, p=.006, \eta^{2}=.159\right]$. Pais da cidade de Erechim $(M=23.72, d p=6.64)$ apresentaram menor nível de estresse em relação aos pais da cidade de Carazinho $(M=31.07, d p=9.02)$ e pais com filhos na faixa etária entre 0 e 3 anos $(M=22.13, d p=6.58)$ apresentaram menor nível de estresse em relação aos pais com filhos na faixa etária entre 13 e 18 anos $(M=30, d p=9.28)$.

0 nível de estresse total apresentou diferenças significativas relacionadas à faixa etária dos filhos $[F(4,85)=3.30, p=$ $\left..014, \eta^{2}=.135\right]$ e à escolaridade dos pais $[F(2,87)=3.38, p=$ $\left..025, \eta^{2}=.081\right]$. Pais com filhos entre 13 e 18 anos $(M=86.56$, $\mathrm{dp}=21.67$ ) apresentaram maior nível de estresse total em relação aos pais com filhos entre 0 e 3 anos $(M=68.40, d p=15.84)$, e pais com escolaridade média $(M=85.08, d p=17.22)$ também apresentaram maior nível de estresse total em relação aos pais com escolaridade superior $(M=72.33, d p=16.70)$. 
Tabela 2. Resultado de medidas em T1 e T2

\begin{tabular}{|c|c|c|c|c|c|c|c|}
\hline & \multirow{2}{*}{ Questionário } & \multirow[t]{2}{*}{$\mathbf{n}$} & \multirow{2}{*}{\multicolumn{2}{|c|}{$\frac{\mathrm{T} 2}{\mathrm{M}(\mathrm{DP})}$}} & \multirow[t]{2}{*}{$\mathbf{F}$} & \multirow[t]{2}{*}{$\mathbf{p}$} & \multirow[t]{2}{*}{$\eta^{2}$} \\
\hline & & & & & & & \\
\hline \multirow{4}{*}{ Apoio Social } & QASC Integração Comunitária & 89 & $17.80(3.56)$ & $17.92(2.82)$ & $(1,87)=1.19$ & .270 & .014 \\
\hline & QASC Participação Comunitária & 81 & $19.74(4.95)$ & $21.14(4.13)$ & $(1,79)=9.36$ & .003 & .106 \\
\hline & QASC AS Informais & 86 & $36.54(5.77)$ & $37.01(5.89)$ & $(1,83)=0.00$ & .960 & .000 \\
\hline & QASC AS Formais & 94 & $15.48(2.39)$ & $15.84(2.36)$ & $(1,92)=0.67$ & .415 & .007 \\
\hline \multirow{6}{*}{$\begin{array}{l}\text { Práticas } \\
\text { Parentais }\end{array}$} & IPP Envolvimento Social & 93 & $20.74(3.90)$ & $21.10(4.48)$ & $(1,92)=.449$ & .504 & .005 \\
\hline & IPP Envolvimento Didático & 93 & $22.48(4.76)$ & $22.79(5.28)$ & $(1,92)=.320$ & .573 & .003 \\
\hline & IPP Envolvimento Disciplina & 92 & $17.02(4.19)$ & $18.47(3.29)$ & $(1,91)=22.1$ & .000 & .196 \\
\hline & IPP Envolvimento Afetivo & 91 & $34.74(5.67)$ & $34.51(6.70)$ & $(1,90)=.079$ & .780 & .001 \\
\hline & IPP Responsabilidade & 91 & 16.89 (3.39) & $16.75(3.71)$ & $(1,90)=.098$ & .755 & .001 \\
\hline & IPP Total & 93 & $111.18(17.15)$ & $113.00(19.42)$ & $(1,92)=.712$ & .401 & .008 \\
\hline \multirow{6}{*}{$\begin{array}{l}\text { Capacidades } \\
\text { dificuldades } \\
\text { dos filhos }\end{array}$} & SDQ Sintomas Emocionais & 88 & $3.12(1.94)$ & $2.31(1.76)$ & $(1,87)=20.2$ & .000 & .189 \\
\hline & SDQ Problemas de Conduta & 87 & $2.09(1.72)$ & $1.68(1.53)$ & $(1,86)=8.24$ & .005 & .087 \\
\hline & SDQ Hiperatividade & 89 & $3.53(2.50)$ & $3.19(2.60)$ & $(1,88)=3.92$ & .051 & .043 \\
\hline & SDQ Problemas com colegas & 88 & $1.54(1.53)$ & $1.36(1.22)$ & $(1,87)=1.42$ & .235 & .016 \\
\hline & SDQ Comportamento Pró Social & 89 & $8.17(1.93)$ & $8.17(1.90)$ & $(1,88)=.000$ & 1.00 & .000 \\
\hline & SDQ Total & 89 & $18.43(5.57)$ & $16.73(4.97)$ & $(1,88)=11.4$ & .001 & .115 \\
\hline \multirow{4}{*}{$\begin{array}{l}\text { Estresse } \\
\text { Parental }\end{array}$} & IEP Distúrbio Parental & 75 & $27.78(6.89)$ & $26.16(6.12)$ & $(1,72)=0.04$ & .834 & .001 \\
\hline & IEP Interação Disfuncional PI & 74 & $23.28(7.24)$ & $22.32(6.15)$ & $(1,69)=0.25$ & .620 & .004 \\
\hline & IEP Criança Difícil & 73 & $27.52(7.72)$ & $25.06(5.91)$ & $(1,70)=0.01$ & .913 & .000 \\
\hline & IEP Estresse Total & 75 & $78.32(19.40)$ & $73.26(15.89)$ & $(1,72)=0.06$ & .803 & .001 \\
\hline
\end{tabular}

\section{DIFERENÇA T1X T2 NOS ESCORES DE QASC, IPP, SDQ E IEP}

Os resultados da diferença entre os dois tempos de avaliação estão apresentados na Tabela 2. Em relação ao QASC, a participação comunitária apresentou diferença significativa e houve um aumento no escore pós-intervenção a respeito da avaliação inicial, sugerindo maior participação dos pais em atividades comunitárias após a participação no Círculo de Debates da EPB. Nos demais itens desse questionário, não foi observada nenhuma diferença significativa.

No IPP, o Envolvimento com a disciplina apresentou diferença significativa, aumentando após a intervenção. Nos demais eixos, houve algumas mudanças não significativas. O SDQ apresentou diferenças significativas na percepção das capacidades/dificuldades dos filhos relacionadas aos sintomas emocionais e aos problemas de comportamento e total, com diminuição no escore pós-intervenção. Além disso, as médias de cada eixo do instrumento nos dois tempos pontuaram dentro de escores considerados normais, não apontando nenhuma dificuldade maior das crianças na percepção dos pais. Já a média total, antes da intervenção, revela dificuldades percebidas dentro de um escore considerado anormal, tendo melhorado, após a intervenção, apresentando um escore limítrofe, mais próximo do normal. Em relação ao IEP, não foram observadas diferenças significativas após a intervenção. As médias dos escores de estresse total se mantiveram dentro de um nível considerado normal de estresse, de acordo com os valores estabelecidos pelos autores do instrumento.

Por fim, foram computados os gradientes de mudança das variáveis QASC Participação Comunitária, IPP Envolvimento com a Disciplina e SDQ total. O resultado da correlação entre tais variáveis não indicou associações significativas (todos os p's >.30), sugerindo que o efeito da ação da EPB é independente em cada um dos desfechos.

\section{DISCUSSÃO}

Este estudo buscou traçar um perfil dos pais/mães que participam dos Círculos de Debates da EPB desenvolvidos em diferentes cidades do Rio Grande do Sul, e avaliar alguns aspectos relacionados ao exercício da parentalidade que podem ser positivamente influenciados por esta participação. 


\section{H. INTERACÃO EM KT PSICOLOGIA}

Com relação ao perfil, predominou a participação de muIheres, o que está de acordo com as demais pesquisas sobre o perfil de participantes de programas de treinamento para pais (Guisso, et al., 2019). Além disso, o público alcançado pelo trabalho da EPB é bastante diversificado, uma vez que não é direcionado a pais de uma faixa etária ou classe social específica.

O primeiro fator avaliado foi o apoio social comunitário. Nesse aspecto, pais com escolaridade mais alta perceberam maior possibilidade de apoio em sistemas informais, como, por exemplo, associações desportivas e culturais e grupos sociais ou religiosos. Quanto à integração e participação comunitária, foram observadas diferenças entre municípios que podem estar relacionadas às comunidades locais, mas também podem decorrer do modo como os grupos se organizaram - se eram grupos de pessoas que já se conheciam e participavam juntos em outras atividades comunitárias ou grupos de pessoas que passaram a se conhecer nos encontros proporcionados pelo Círculo de Debates, por exemplo. Além disso, houve um aumento do escore de participação comunitária após a intervenção, podendo indicar uma satisfação por participar do Círculo de Debates, valorizado como espaço que possibilitou a construção de relações de apoio social e comunitário, fator chave no desenvolvimento social e familiar. De acordo com Álvarez et al. (2019), a qualidade da rede social dos pais deve ser avaliada e aprimorada como fator preventivo para as famílias em risco, uma vez que foi um fator promotor de melhores resultados do programa.

A respeito das práticas parentais, constatou-se um maior envolvimento relatado pelas mães em relação aos pais em três eixos (envolvimento didático, envolvimento afetivo e responsabilidade) e no geral (total). Tal resultado indica que as mães desta pesquisa se mostraram mais envolvidas com seus filhos, resultado também encontrado por Grzybowsky e Wagner (2010) em uma pesquisa sobre o envolvimento parental após a separação. Os pais de crianças de 0 a 3 anos relataram maior envolvimento social com os filhos em comparação aos pais de adolescentes de 13 a 18 anos. Tal fato pode estar relacionado às questões próprias de cada faixa etária, em que a necessidade de envolvimento parental e a vida social são muito diferentes. Os pais do município de Erechim relataram maior envolvimento social com os filhos em relação aos pais do município de Casca, fator que pode estar relacionado à faixa etária dos filhos, como também aos tipos de atividades sociais disponíveis em cada cidade (a primeira de médio e a segunda de pequeno porte).

Os pais com escolaridade superior relataram maior envolvimento com a disciplina em relação aos pais com escolaridade fundamental, demonstrando que a escolaridade influencia na forma de estabelecer e cobrar regras, colocando limites e castigos. Um maior envolvimento foi constatado também em relação aos pais com filhos de 0 a 3 anos em relação aos
Bruna Detoni, Adriane Xavier Arteche e Adolfo Pizzinato

pais de filhos de 7 a 12 anos. Pais mais envolvidos nesse eixo usam menos gritos e castigos físicos, conseguindo colocar limites de forma mais afetiva.

Houve aumento significativo no envolvimento com a disciplina após a intervenção, o que pode indicar que a participação no Círculo de Debates auxilia os pais a buscarem melhores formas de disciplina na educação dos filhos, já que aborda a importância do estabelecimento de regras claras e limites, sempre com afeto e respeitando a maturidade das crianças. Tal resultado também foi encontrado por Álvarez et al. (2018), que referiu que os pais diminuíram o uso de castigos corporais após a participação no Programa Crecer Felices en la Família. Guisso et al. (2019) referem que pais que participaram de programas de treinamento indicaram satisfação com a participação, além de terem conseguido mudar algumas práticas que refletiram na melhora de determinados comportamentos dos filhos. Muitos desses pais recomendaram a participação de outros pais nos programas, acreditando que esse espaço proporcionou trocas e reflexões em relação às posturas que precisam ser reavaliadas em sua interação com os filhos.

Com relação à percepção das capacidades e dificuldades dos filhos, os sintomas de hiperatividade foram mais relatados por pais mais jovens e percebidos mais em meninos, o que pode estar relacionado à faixa etária dos filhos e ao fato de as crianças estarem sendo percebidas como muito mais hiperativas atualmente, havendo outros estudos que corroboram a percepção de que os meninos são mais ativos do que as meninas (Cardoso et al., 2007). A escolaridade mais baixa dos pais foi fator importante para o relato de maiores dificuldades dos filhos, relacionadas aos sintomas emocionais e aos problemas com os colegas. Pais com maior escolaridade são mais motivados, menos estressados e mais satisfeitos com o comportamento dos filhos (Guisso et al, 2019).

Mudanças significativas foram observadas a respeito da percepção dos pais sobre as capacidades e dificuldades dos filhos após a participação no Círculo de Debates, principalmente a respeito de sintomas emocionais, problemas de comportamento, hiperatividade e total. Essa mudança pode estar relacionada ao fato de dois encontros estarem destinados a discutir marcos importantes do desenvolvimento típico, ou seja, conhecer o que é esperado no desenvolvimento da infância e da adolescência. Dessa forma, o que, na avaliação pré-intervenção, era percebido como um sintoma, pode ter sido relacionado a algum fato do desenvolvimento típico e já não foi relatado depois. Álvarez et al. (2018) referem que pais que completaram o Programa Crecer Felices en la Família passaram a ter uma probabilidade significativamente menor de construir expectativas inapropriadas em relação à criança e a responder mais empaticamente aos filhos. 


\section{MLE INTERACÃO EM PSICOLOGIA}

Relativamente ao estresse parental, no Eixo Distúrbio Parental, as mães apresentaram maior estresse do que os pais, o que pode estar relacionado às múltiplas tarefas que elas ainda desempenham atualmente, o que as faz sentirem-se mais sobrecarregadas. Quanto à Interação Disfuncional Parental Infantil, houve um relato de maior interação disfuncional de pais com crianças na faixa etária de 7 a 18 anos, período em que os pais precisam acompanhar as atividades (escolares e extracurriculares) dos filhos, de entrada e passagem pela adolescência, quando os conflitos são mais comuns. Pais com baixa escolaridade também indicaram maior interação disfuncional com seus filhos, apontando um fator a ser considerado. No Domínio Criança Difícil, também os pais relataram os filhos de 13 a 18 como mais difíceis em relação aos bebês, sugerindo que a fase da adolescência é vivida com mais estresse.

Respectivamente ao estresse total, considerando que ele reflete tensões que ocorrem em áreas de angústia pessoal, decorrentes da interação com os filhos, e outras que surgem das características comportamentais das crianças (Minetto et al., , Crepaldi \& Bigras, 2012), os pais com Ensino Superior de crianças na faixa etária de 0 a 3 anos apresentaram estresse considerado normal, já os pais com Ensino Médio e filhos entre 13 e 18 anos apresentaram níveis de estresse considerados elevados. Os participantes não manifestaram mudanças significativas relacionadas ao estresse parental ( $T 1 \times T 2)$, o que pode indicar que a medida de estresse está ligada a outros fatores da vida desses pais e mães não avaliados neste estudo.

Dessa forma, é possível inferir que o trabalho desenvolvido no Círculo de Debates da EPB tem um impacto positivo no exercício da parentalidade de pais e mães, principalmente relativo ao apoio social e comunitário, tornando-se um espaço importante de trocas de experiências e fazendo com que tenham uma melhor percepção das capacidades e dificuldades dos filhos. Isso colabora positivamente com o desenvolvimento dos filhos, sobretudo na ampliação de percepção das potencialidades das crianças, e promove formas mais responsivas e assertivas de lidar com dificuldades de conduta. As correlações sugeriram que o efeito dessa ação da EPB é independente em cada um dos desfechos, ou seja, as mudanças observadas na participação comunitária podem ser fruto do formato do trabalho, com grupos de pais e trocas de experiências, caracterizando esse espaço pela participação comunitária, já que é uma iniciativa fomentada e integrada por membros de suas comunidades. A melhor percepção das capacidades e dificuldades dos filhos e o envolvimento com a disciplina de forma mais positiva podem estar relacionadas ao conhecimento do conteúdo trabalhado nos encontros e, possivelmente, ter influenciado a percepção comunitária dos integrantes, especialmente dos seus facilitadores. Programas que buscam auxiliar mães e pais a aprenderem novas habilidades em relação à socialização
Bruna Detoni, Adriane Xavier Arteche e Adolfo Pizzinato

dos filhos e à imposição de limites para determinados comportamentos são maneiras preventivas de torná-los mais seguros, com melhores estratégias para lidar com os desafios cotidianos (Guisso et al., 2019).

A despeito da relevância dos resultados, convém ressaltar que o presente estudo teve como viés o fato de os grupos acontecerem em cidades diferentes, com difícil acesso para aplicar os questionários. Por outro lado, seria importante poder pesquisar outros estados e fazer estudos comparativos, de modo a avaliar mais amplamente a eficácia de um programa social dessa natureza, considerando inclusive aspectos culturais. Sugere-se ainda que outros estudos possam analisar outras variáveis relacionadas às práticas parentais positivas, como o senso de competência parental, que avalia satisfação e eficácia parental (To et al., 2014; Reedtz et al., 2010) e indicadores de saúde mental parental. Também é interessante poder realizar testagem follow up com o objetivo de avaliar os pais depois de um tempo mais longo da intervenção, uma vez que algumas mudanças de comportamento e práticas parentais podem demorar mais tempo para acontecer.

Este estudo procurou demonstrar o impacto de programas de promoção de práticas parentais positivas para o desenvolvimento infantil e comunitário. Além disso, destacou a importância e a necessidade de existirem mais programas que promovam tais práticas. Ao serem oferecidos a pais, mães e cuidadores de crianças, em parceria com outras instituições (públicas ou privadas), eles potencializam seus efeitos e podem se transformar em objeto de pesquisa e estudo.

\section{DECLARAÇÃO DE CONFLITOS DE INTERESSES}

Os autores declaram que não há conflitos de interesses no presente artigo.

\section{REFERÊNCIAS}

Álvarez, M.; Rodrigo, M. J.; Byrne, S. (2018). What implementation componentes predicts outcomes in a parenting program? Research on Social Work Practice, 28(2), 173-187. https://doi.org/10.1177/1049731516640903.

Álvarez, M.; Byrne, S.; Rodrigo M. J. (2019). Patterns of individual change and program satisfaction in a positive parenting program for parents at psychosocial risk. Child \& Family Social Work, 25(2), 230-239. https://doi.org/10.1111/ cfs. 12678.

Barroso, R. G.; Machado, C. (2010). Definições, dimensões e determinantes da parentalidade. Psychologica, (52-I), 211229. https://doi.org/10.14195/1647-8606_52-1_10. 


\section{H. INTERACÃO EM LFICOLOGIA}

Benetti, S. P. da C.; Balbinotti, M. A. A. (2003). Elaboração e estudo de propriedades psicométricas do Inventário de Práticas Parentais. Psico-USF, 8(2), 103-113. https://doi. org/10.1590/s1413-82712003000200002.

Belsky, J. (1984). The Determinants of Parenting: a Process Model. Child Development, 55(1), 83-96. https://doi. org/10.2307/1129836.

Brito, A.; Faro, A. (2016). Estresse Parental: Revisão sistemática de estudos empíricos. Psicologia em Pesquisa, 10(1), 64-75. https://doi.org/10.24879/201600100010048.

Cardoso, F. L.; Sabbag, S.; Beltrame, T. S. (2007). Prevalência de transtorno de déficit de atenção/hiperatividade em relação ao gênero de escolares. Revista Brasileira de Cineantropometria \& Desenvolvimento Humano, 9(1), 50-54.

Dezoti, A. P. O.; Alexandre, A. M. C.; Tallmann, V. A. B.; Maftum, M. A.; Mazza, V. de A. (2013). Rede social de apoio ao desenvolvimento infantil segundo a equipe de saúde da família. Escola Anna Nery, 17(4), 721-729. http://dx.doi. org/10.5935/1414-8145.20130017.

Escola de Pais do Brasil. (2021). Retirado de: http://escoladepais.org.br/cursos/presenciais/.

Gracia, E.; Herrero, J.; Musitu, G. (2002). Evaluación de recursos y estresores psicosociales en la comunidad. Síntesis.

Grzybowski, L. S.; Wagner, A. (2010). O Envolvimento Parental Após a Separação/Divórcio. Psicologia: Reflexão e Crítica. 23(2), 289-298. https://doi.org/10.1590/s010279722010000200011.

Guisso, L.; Bolze, S. D. A.; Viera, M. L. (2019). Práticas parentais positivas e programas de treinamento parental: uma revisão sistemática da literatura. Contextos Clínicos, 12(1) https://doi.org/10.4013/ctc.2019.121.10.

Houzel, D. (2004). As implicações da parentalidade. In: L. Solis-Pontos (Org.). Ser pai, ser mãe: Parentalidade: um desafio para o terceiro milênio [M. C. P. da Silva, trad.] (pp. 47-51). Casa do Psicólogo.

Lamela, D.; Castro, M.; Gonçalves, T.; Figueiredo, B. (2012). "PApi - Pais por inteiro" Programa de intervenção em grupo para o ajustamento e a promoção da coparentalidade positiva em pais divorciados. Análise Psicológica, 27(4), 493-507. https://doi.org/10.14417/ap.243.

Macana, E. C.; Comim, F. (2015). O papel das práticas e estilos parentais no desenvolvimento da primeira infância. In: G. A. Pluciennik, M. C. Lazzari, \& M. F. Chicaro. (Orgs.) Fundamentos da Família como Promotora do Desenvolvimento Infantil: Parentalidade em Foco (pp. 34-47). Fundação Maria Cecília Souto Vidigal. https://www.fmcsv.org.br/pt-BR/ biblioteca/fundamentos-da-familia/.

Macoby, E. E. (2000). Parenting and its effects on children: on reading and misreading behavior genetics. Annual Review of Psychology, 51: 1-27. https://doi.org/10.1146/annurev. psych.51.1.1.
Minetto, M. F.; Crepaldi, M. A.; Bigras, M.; Moreira, L. C. (2012). Práticas Educativas e estresse parental de pais de crianças pequenas com desenvolvimento típico e atípico. Educar em Revista, 43, 117-132. https://doi.org/10.1590/ s0104-40602012000100009.

Pereira, L. M.; Viera, C. S.; Toso; B. R. G. O.; Carvalho, A. R. S.; Bugs, B. M. (2016). Validação da escala de Índice de Estresse Parental para o português do Brasil. Acta Paulista de Enfermagem, 29(6), 671-677. https://doi.org/10.1590/19820194201600094.

Pinho-Pereira, S.; Vestana, C. B.; Costa-Lobo, C. (2017). Parentalidade Positiva e Bem-Estar Subjetivo: Intervenção com Pais de Estudantes Sobredotados. Revista de Estudios e Investigación en Psicología y Educación, vol. Extr.(5). https://doi.org/10.17979/reipe.2017.0.05.2954.

Reedtz, C.; Handegard, B. H.; Mørch, W. T. (2011). Promoting positive parenting practices in primary care: outcomes and mechanisms of change in a randomized controlled risk reduction trial. Scandinavian Journal of Psychology, 52(2), 131-137. https://doi.org/10.1111/j. 1467-9450.2010.00854.x.

Rodrigo, M. J. (2016). Quality of implementation in evidence-based positive parenting programs in Spain: Introduction to the special issue. Psychosocial Intervention, 25(2), 6368. https://doi.org/10.1016/j.psi.2016.02.004.

Saur, A. M.; Loureiro, S. R. (2012). Qualidades psicométricas do Questionário de Capacidades de Dificuldades: uma revisão de literatura. Estudos de Psicologia (Campinas), 29(4), 619-629. https://doi.org/10.1590/s0103$166 \times 2012000400016$.

Schmidt, B.; Staudt, A. C. P.; Wagner, A. (2016). Intervenções para promoção de práticas parentais positivas: uma revisão integrativa. Contextos Clínicos, 9(1), 2-18. https://doi. org/10.4013/ctc.2016.91.01.

To, S., So, Y.; Chan, T. (2014). An exploratory study on the effectiveness and experience of a parent enhancement group adopting a narrative approach. Journal of Social Work, 14(1), 41-61. https://doi.org/10.1177/1468017313475554.

Weber, L. N. D.; Brandenburg, O. J.; Salvador, A. P. V. (2006). Programa Qualidade na Interação Familiar (PQIF): Orientação e Treinamento para Pais. Psico, 37(2), 139-149. 